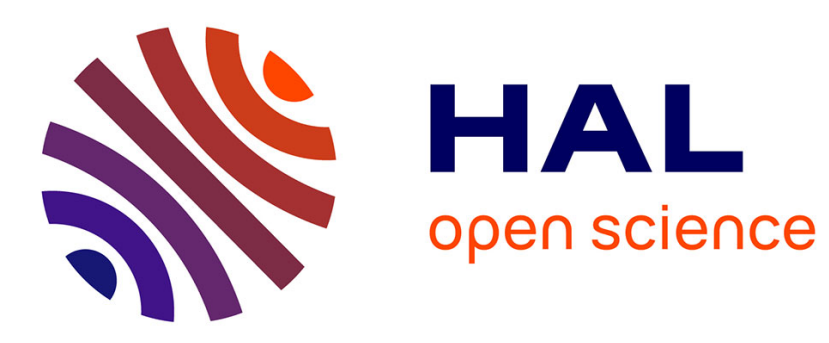

\title{
Prediction of Drying Indices for Paddy Rice in a Deep Fixed-Bed Based on Neural Network
} Danyang Wang, Chenghua Li, Benhua Zhang, Ling Tong

\section{To cite this version:}

Danyang Wang, Chenghua Li, Benhua Zhang, Ling Tong. Prediction of Drying Indices for Paddy Rice in a Deep Fixed-Bed Based on Neural Network. 10th International Conference on Computer and Computing Technologies in Agriculture (CCTA), Oct 2016, Dongying, China. pp.496-507, 10.1007/978-3030-06155-5_51. hal-02179974

\section{HAL Id: hal-02179974 \\ https://hal.inria.fr/hal-02179974}

Submitted on 12 Jul 2019

HAL is a multi-disciplinary open access archive for the deposit and dissemination of scientific research documents, whether they are published or not. The documents may come from teaching and research institutions in France or abroad, or from public or private research centers.
L'archive ouverte pluridisciplinaire HAL, est destinée au dépôt et à la diffusion de documents scientifiques de niveau recherche, publiés ou non, émanant des établissements d'enseignement et de recherche français ou étrangers, des laboratoires publics ou privés. 


\title{
Prediction of Drying Indices for Paddy Rice in a Deep Fixed-bed Based on Neural Network
}

\author{
Danyang Wang ${ }^{1}$, Chenghua $\mathrm{Li}^{2(\bowtie)}$, Benhua Zhang ${ }^{1}$, Ling Tong ${ }^{1}$ \\ ${ }^{1}$ College of Engineering, Shenyang Agricultural University, Shenyang, China \\ wdy@syau.edu.cn, \{ benhuazhang, gc.tongling\}@163.com \\ ${ }^{2}$ College of mechanical Engineering, Shenyang Ligong University, Shenyang, China \\ lch@mail.sylu. edu.cn
}

\begin{abstract}
In this study, four artificial neural network models are developed for paddy rice drying in a deep fixed-bed to predict five drying performance indices, including additional crack percentage, drying moisture uniformity, energy efficiency rate, germinating percentage and drying time. The four neural networks are BP, RBF, GRNN and ELMAN. After plenty of trials with a variety of neural network architectures, neural network with five inputs and five outputs is better than network with five inputs and any other outputs. Five drying parameters including paddy original moisture content, air temperature, air velocity, paddy thickness and tempering time are regarded as input vectors of the neural networks. The experimental results show that neural networks have good performance in predicting the paddy drying process. And also, the simulation indicate that the RBF neural network has advantages over other three neural networks in performance.
\end{abstract}

Keywords: Neural network · Prediction · Drying indices · Paddy rice · Deep fixed-bed drying · Drying parameters

\section{Introduction}

Paddy rice drying is a processing technique to low down the moisture content of the original harvest paddy. For grains, drying is a prerequisite to for further processes like storage and processing, hence it is of great importance in grain production. High quality of paddy not only reduces the postharvest losses, but also improves the eating quality and economic efficiency. Typically, paddy drying is an important sign to show the level of a country's mechanized commissariat. For drying quality and drying technology, there are several influencing factors, including additional crack percentage, energy consumption rate, germination rate, drying time, drying moisture uniformity. 1Paddy drying is a complex heat and mass transfer process, which includes preheating, constant rate of drying, decreasing rate of drying, tempering and cooling. Since these factors and processes have complex interaction rules among them, analyzing the effect rules of the drying process becomes a hard work.

However, Artificial Neural Network (ANN) provides a new and effective way to model the process of paddy drying. ANNs are used to be solutions for various problems in scientific research and engineering problems, especially for some situations when the conventional modeling methods are not applicable (Zaidi et al., 1999). As a data-processing system ANN is originated from biological neural system and well

Biography: Wang Danyang: Doctor, College of Engineering, Shenyang Agricultural University, Shenyang , China 110866; Research field: Agricultural products engineering. Email: wdy@syau.edu.cn

${ }^{\bowtie}$ Corresponding author: Li Chenghua : Doctor, Professor, College of mechanical Engineering, Shenyang Ligong University, Shenyang , China 100168; Research field: Agricultural Mechanization Engineering.Email: lch@mail.sylu. edu.cn 
trained ANNs should be regarded as the preferred model for its better prediction. The predictive power of an ANN is conditional on the training results of sample data and on the verification of new independent data observed. When there is new data available, the artificial neural network can improve its performance because of its relearn ability. Artificial neural network model is developed based on a multi-input variable and multi-output variable structure. Unlike traditional modeling methods, its modeling is not dependent on heuristic knowledge of simulation mapping relations (Ramesh et al., 1996). Farkas et al. (2000) established a neural network model for the characteristic index prediction of grain drying. Jiang et al. (1999) used artificial neural networks to predict winter wheat harvest. Zhang et al. (2000) applied neural networks to harvesting and drying techniques. Compared with the traditional simulation methods, the use of artificial neural network model for prediction usually has a faster processing speed. Furthermore, the input and output variables of the artificial neural network can be added and deleted according to the actual situation. The purpose of this study is to compare and select a better artificial neural network for the performance prediction of rice drying by using the collected test data. The optimal goal of paddy drying is to obtain better product quality with minimum energy consumption, so the real-time on-line control of drying process is very important. Compared with the traditional mathematical model, the artificial neural network is faster, because it does not need complex iterative calculation to solve the differential equation. In artificial neural networks, the results can be obtained by simple multiplication of the input and the weight matrix. (Zhang,2002).

The outline and organization of the rest of this paper is as follows. Section 2 recommends the research of paddy rice drying experiment. Then four kinds of ANN models are presented in Section 3. And also, comparisons of the predicting results of four different ANNs are presented. Finally, there are some conclusions in Section 4.

\section{Experiment}

six performance indices and Five drying parameters are in the prediction model for paddy rice drying in a deep-fixed bed. The five drying parameters are original moisture

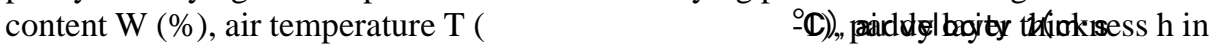
$\mathrm{m}$, tempering time te (min). These five parameters used as the inputs of the ANNs mentioned in this paper. The five performance criteria for assessing the drying process are additional crack percentage $\mathrm{C}(\%)$, drying duration td (min), drying moisture uniformity D, germinating percentage G $(\%)$, energy efficiency rate $E(\mathrm{~kJ} \bullet \mathrm{kg}-1 \bullet \mathrm{h}-1)$ and they are select as the outputs of the ANNs mentioned in this paper..

The experiments which conducted for paddy rice drying in a fixed-bed dryer use a paddy cultivar named 'Liaojing 294' mainly planted in Liaoning Province with original moisture contents $(12 \%$

and relative humidity ranges from 54\% fizerd .bed deap used for analyzing five factors by using the quadratic regression orthogonal rotary experiments [5].

The structure of the deep-fixed bed is as fig.1. Coming from the air compressor of the system, the airflow with stable pressure flows through the control valve and the air flow meter, and then the air flow transported to the drying chamber to dry the inside-materials after it is heated by the heater. There are some test holes in the drying chamber, which are used for measuring temperature, humidity and other parameters of each paddy layer. In each drying experiment, moisture contents of each test point in each grain-layer will be obtained through weight measurement of each test point by a special sampler once all the moisture contents of test points come up to the safe storage standard $14 \%$ (w.b.). Each factor coding level for quadratic orthogonal rotation experiments were determined as shown in Table 1. 


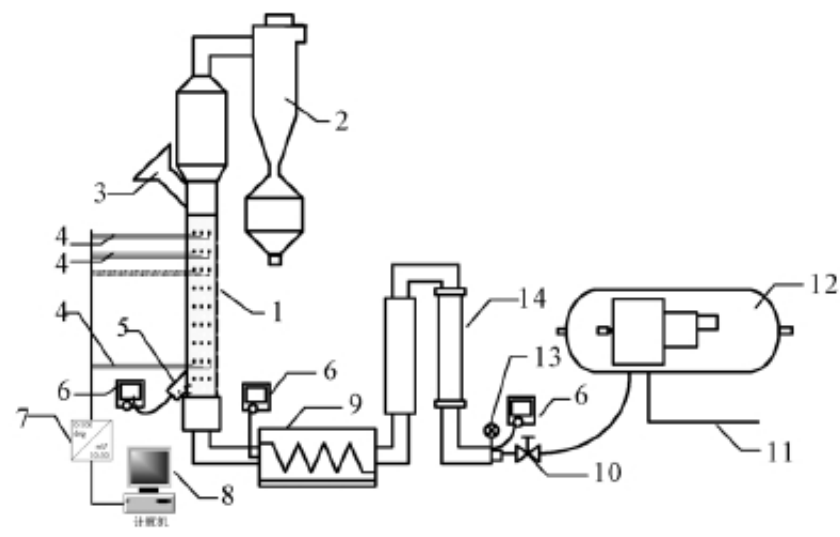

1. Drying chamber 2. Rotary separater 3. Feed inlet 4. Sensors 5. Material discharge 6. Electronic digital temperature measurer 7. Mcusystem 8. Master computer 9. Heater 10. Control valve 11. Exhaust pipe for stable pressure 12. Air compressor 13. Pressure gauge 14. Rotameter

Fig. 1. Structure of drying test bed

Table. 1. Coding of factor level

\begin{tabular}{cccccc}
\hline$x_{\mathrm{j}}$ & $\begin{array}{c}X_{1} /{ }^{\circ} \mathrm{C} \\
\text { Air } \\
\text { temperature }\end{array}$ & $\begin{array}{c}X_{2} / \mathrm{ms}^{-1} \\
\text { Air } \\
\text { velocity }\end{array}$ & $\begin{array}{c}X_{3} / \mathrm{cm} \\
\text { Paddy } \\
\text { thickness }\end{array}$ & $\begin{array}{c}X_{4} / \% \\
\text { Original } \\
\text { moisture } \\
\text { content }\end{array}$ & $\begin{array}{c}X_{5} / \mathrm{t} \\
\text { tempering } \\
\text { time }\end{array}$ \\
\hline+2 & 85 & 1.20 & 60 & 28 & 6 \\
+1 & 75 & 1.01 & 50 & 25 & 4.5 \\
0 & 65 & 0.83 & 40 & 22 & 3 \\
-1 & 55 & 0.64 & 30 & 19 & 1.5 \\
-2 & 45 & 0.45 & 20 & 16 & 0 \\
\hline
\end{tabular}

In the following we introduce the measurement methods of the indices:

(1) Measurement of moisture content

Two measurement methods of rice moisture content are used in this paper: the standard oven method and difference measurement of rice weight method. The original moisture content of the paddy rice is measured via the oven method $\left(5 \mathrm{~g}, 105^{\circ} \mathrm{C}, 24 \mathrm{~h}\right)$ and get an average of the five tests. In each drying experiment, moisture content of each test point in each grain-layer will be obtained through weight measurement of each test point by a special sampler every certain interval until all the moisture contents of test points come up to the safe storage standard $14 \%$ (w.b.). In order to ensure an effective data collection, time sampling interval was set as follow, the earliest $5 \mathrm{~min} \sim 10 \mathrm{~min}$, initial stage $15 \mathrm{~min}$

, thte BOddre and later period $20 \mathrm{~min}$

(2) Measurement of rice temperature

During the drying process, a temperature auto-measuring system was used and synchronously records time and weight of rice sample.

(3) Measurement of drying evenness

Eight parameter layers were set according to the distance to the bottle of drying chamber, respect layers are $30 \mathrm{~cm}, 60 \mathrm{~cm}, 90 \mathrm{~cm}, 120 \mathrm{~cm}, 150 \mathrm{~cm}, 250 \mathrm{~cm}, 330 \mathrm{~cm}, 410 \mathrm{~cm}$. Each layer has three measure points, and distance of each point to the cross section of the circle was separately $1 / 6 \mathrm{R}, 1 / 2 \mathrm{R}$ and $1 / 3 \mathrm{R}$, positions of layers and points were shown as fig.2.

According to the regulation of “Test Method of Grain Dryer” (GB6970-1986), for drying moisture un-uniformity, no less than 5 points at different positions on the cross-section must be selected to measure its respect moisture content after drying. The 
drying moisture un-uniformity equals to the difference of maximum and minimum moisture content. This definition may reflect the maximum difference of paddy rice moisture content in the drying chamber, but it is more sensitive to the discretization of statistical data, and cannot comprehensively reflect the whole situation of paddy rice moisture content after drying. Therefore in this work, paddy moisture content of all measured points in each layer were measured at the final moment of drying, and then all these values were chosen as sub sample of drying moisture uniformity, drying moisture uniformity $D$ is the reciprocal of the standard deviation of sample set, as shown in the following equation:

$$
D=1 / \sqrt{\frac{1}{N} \sum_{i=1}^{n} \sum_{j=1}^{m} W_{i j}^{2}-\left(\frac{1}{N} \sum_{i=1}^{n} \sum_{j=1}^{m} W_{i j}\right)^{2}}
$$

where $N$ is the total number of measured points $\mathbb{N} \geqslant 9$ ) $n$ denotes the number of measured layers; $\mathrm{m}$ represents the number of measured points in every layer; $W_{i j}$ indicates the immediate paddy moisture content of the $j$ point in the $i$ th layer.

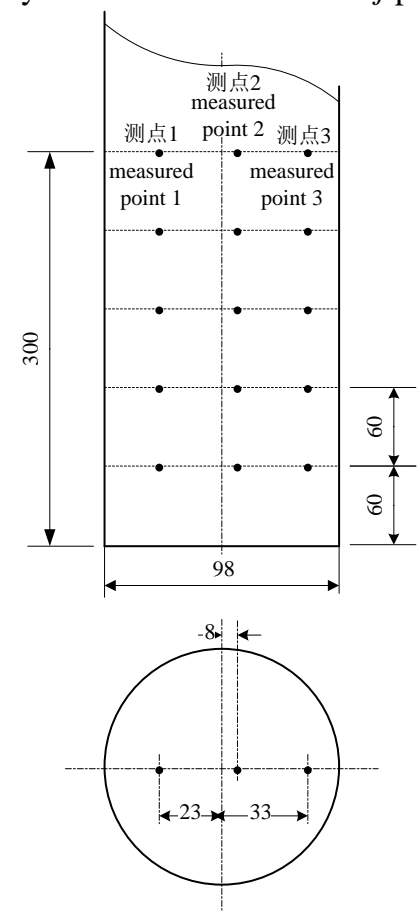

Fig. 2. Positions of measuring points

(4) Germination rate determination

500 grains were randomly selected before the experiment, after 30 minutes soaking in the water $\left(30^{\circ} \mathrm{C}\right)$, all grains were divided into five groups and put in a petri dish with wet cotton yarn, and placed in an environment with a temperature range $20^{\circ} \mathrm{C} \sim 25^{\circ} \mathrm{C}$. Then, the experimental paddy is immersed once a day, 7 days later we measure the germination rate of each group. The average germination rate is $\mathrm{G}_{0}=97.5 \%$ and we take it as the initial germination rate. After drying process, we randomly measure the germination rate of each paddy layer.

(5) Measurement of Drying Time

When air velocity and temperature are regulated as the predetermined value and kept stable, the paddy can be put into the drying cylinder and timing operation is started. The drying process and timing operation is terminated once the paddy moisture content (w.b.) is lower than $14 \%$. The obtained time duration is the total drying time $T_{t}$, which 
equals to the addition of the tempering time and the net paddy drying time $T_{p}$.

(6) Measurement of Energy Consumption

The total energy consumption consists of two parts: energy consumption of air compressors and the one of air heaters. According to the drying technology, the thermodynamic system of drying cylinder is known as an opened thermodynamic system. The drying cylinder of each experiment was divided into 3-5 layers according to the thickness of paddy rice. During the drying process, energy consumption of each layer in different time was determined by the measurement of hot air temperature and moisture contents of paddy rice in each layer. Then, the total energy consumption of each experiment was just the cumulative energy sum of each layer. According to the first law of thermodynamics, the obtained calculated total energy consumption is $Q$. Then, the energy consumption rate can be calculated by

$$
E=Q / \sum_{j=1}^{i} \int_{(r)} \delta_{i} w \cdot \frac{1}{t}
$$

Where $\int_{(r)} \delta_{i} w$ (kg) is evaporation of each layer in drying process and $t(\mathrm{~h})$ is drying time.

(7) Measurement of Additional Crack Percentage

Before the drying process, 300 grains of paddy are randomly selected from original paddy sample and every 100 grains constitute a group. The dried paddy is carefully manually shelled, subsequently the additional crack percentage are detected under a self-made detector lamp ${ }^{[1]}$. The paddy will be judged to be cracked if any cracks are detected. 48 hours after the drying process, the additional crack percentage are finally tend to be stable (Kunze,1975

; Sharma, 1982). Therefore, 48

randomly selected 50 grains from each paddy layer and measure the final additional crack percentage. The difference of the average final additional crack percentage and the original additional crack percentage is the increasing additional crack percentage $C(\%)$.

\section{ANN Prediction Models}

\section{1 BPNN Model}

ANN is a computational methodology which has multi-factor analysis. Each artificial neural network model contains some basic computing nodes, which is used to calculation of nonlinear sum the model with three-layer feed forward and back propagation is the ANN model being most commonly used, known as BPNN model. The topology architecture of a three-layer BPNN model is shown in Fig.3. It is consisted by one hidden layer, one input and one output layer, denoted as $\mathrm{Xi}, \mathrm{Bj}$, and Yk, respectively. There are mutual connections between the nodes of different layers, and there are no connections between the nodes of the same layer. Wij and Wjk denote the connection weights between $\mathrm{Xi}$ and $\mathrm{Bj}, \mathrm{Bj}$ and $\mathrm{Yk}$, respectively.

The prediction model of BPNN contains positive feedback of a data stream and reverse feedback of error signal. In predicting problems, BPNN model must be trained by prior data. During the training, the input nodes receive the data to be processed by the network, and the output layer nodes hold the computation results. All synaptic weights are updated once in each iterative learning process. The training process will not be terminated until the networks error $E$ is lower than the preset error. 


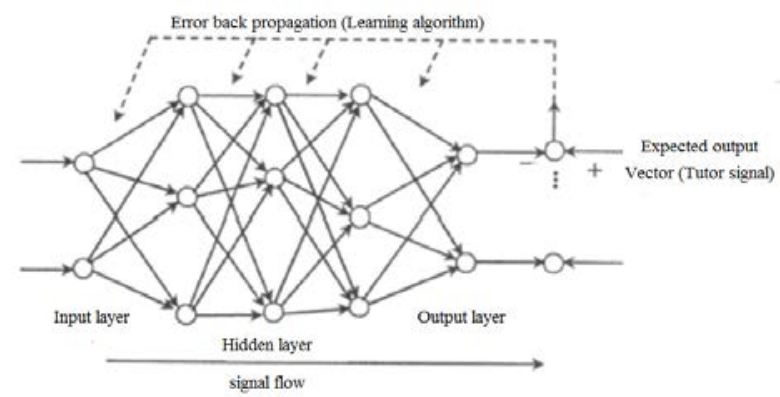

Fig. 3. Structure of BP neural network

Assuming that the network has $h$ inputs and $m$ outputs nodes. $T_{k}$ denotes the desired output. $\theta_{k}, \Theta_{j}$ are the unit thresholds of the output layer and the hidden layer respectively. $f(x)=\frac{1}{1+e^{-x / q}}$ is the active function (q denotes the parameter of sigmoid, $q \in(0,1))$.

\section{2 RBFNN Model}

Radial Basis Function (RBF) is a kind of forward network constructed on the basis of function approximation theory. The study of this kind of neural network is to search the best fitting curved surface for the training data in multidimensional space. The neuron transfer function of each hidden layer of the radial basis function network forms a basic function of the fitting plane. RBF is a kind of local approximation network, that is, there are only a few neurons in the local area of the input space to decide the output of the network.

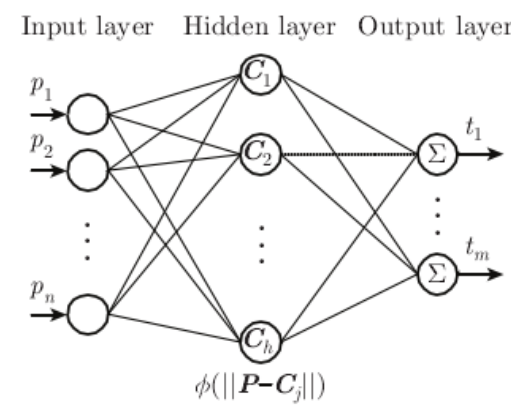

Fig. 4. RBF Neural Network Structure

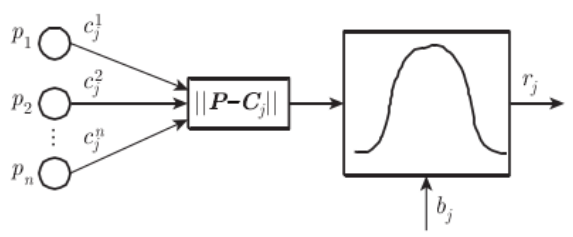

Fig. 5. Structure of the RBF with $n$ inputs

RBF neural network composes three layers, it including the hidden layer, input layer and output layer. The amount of neurons in hidden layer is up to the specific circumstances of problems. As shown in Figure 4 and Figure 5. For each input and output data combination, only a few weights need to be adjusted, so that the network has the advantage of fast learning speed. It has been confirmed that the RBF network can gain upon single-valued continuous function willfully with any degree of accuracy provided that the number of neurons in hidden layer is enough.

\section{3 GRNN Model}


Generalized Regression Neural Network (GRNN) is a subfield of RBF network, which is an extension of probabilistic neural network. This type of network is developed on the base of mathematical and statistical method, GRNN can reflect the implicit mapping relationship in accordance with the sample book. Even though the sample book is not insufficient, its output can also get the optimal constringency of regression and the learning algorithm of GRNN is simple and it has a highly parallel structure. As Figure 6 shows, GRNN structure including the input layer, model layer, sum layer and output layer.

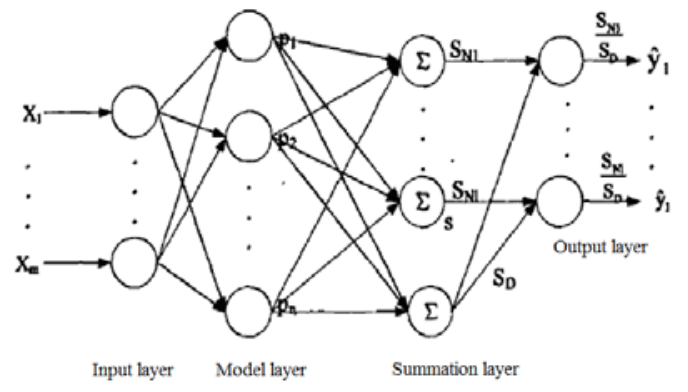

Fig. 6. GRNN Neural Network Structure

Regularization theory is used in the learning algorithm of GRNN; it adds a complex term (regularization term) which is used to limit the approximation function on the basis of the standard error term. The regularization term reflects the "geometric property" of the approximation function. That is:

$$
E_{R}(F)=\frac{1}{2}\|D F\|^{2}
$$

\section{4 ELMAN Model}

For achieving the purpose of memory, Elman network increase a network layer in hidden layer of a feed forward network, therefore the system is able to get with the time varying characteristics, it can also report the characteristics of dynamic process directly. As Figure 5 shows, beside the normal input layer, hidden layer and output layer in the neural structure, there is a special unit, which named state layer. State layer receives the return signal from hidden layer, which let it to be tactful to the historical data. The ELMAN network with internal feedback enhances the ability to process the dynamic information and realize dynamic modeling.

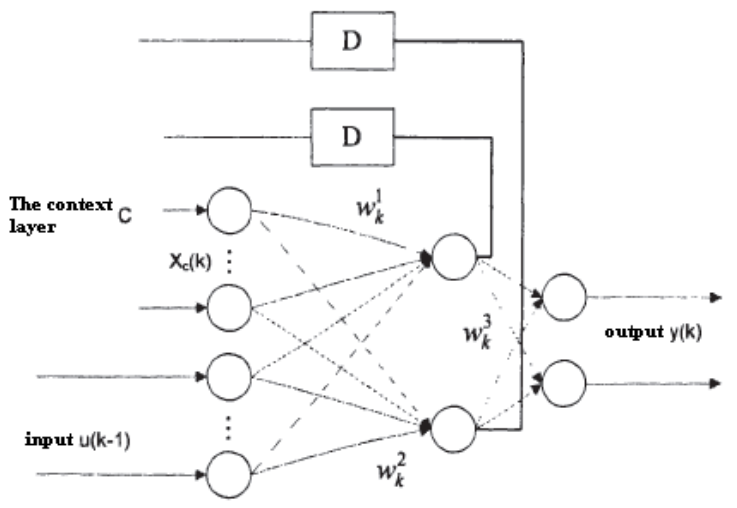

Fig. 7. Elman Neural Network Structure

The external input of Elman neural network learning algorithm is u (k-1), the output is y (k), 
the output of hidden layer is $\mathrm{x}(\mathrm{k})$, so nonlinear state space equations are as follows:

$$
\begin{aligned}
& x(k)=f\left[w_{k}^{1} x_{c}(k)+w_{k}^{2} u(k-1)\right. \\
& x_{c}(k)=a x_{c}(k-1)+x(k-1) \\
& y(k)=g\left[w_{k}^{3} x(k)\right]
\end{aligned}
$$

Where $f$ and $g$ are the transfer functions of the hidden and the output layer respectively, $w_{k}^{1}$ is the connection weight matrix from the state layer to the hidden layer, $w_{k}^{2}$ is the connection weight matrix from the input layer to the hidden layer, $w_{k}^{3}$ is the connection weight matrix from the hidden layer to the output layer. $0 \leq a \leq 1$ is the feedback gain factor of self-connection.

\section{5 Prediction Model of Paddy Rice Drying}

A reasonable setting of the influencing parameters is always the key and difficult problem in paddy drying process. The drying of paddy is mainly effected by air temperature, air velocity, paddy layer thickness, original moisture content and tempering time. Considering the actual application requirements, we develop prediction models based on the four ANNs, and all of them have five inputs and five outputs, as shown in table 1.

Tab. 2. Inputs and outputs of the Neural Network Models

\begin{tabular}{ccc}
\hline & Input & Output \\
\hline Variables & $T, v, W, h, t_{\mathrm{e}}$ & $D, t_{\mathrm{d}}, C, G, E$ \\
\hline
\end{tabular}

The four neural networks have all been trained by 1000 randomly selected samples and the training has all reached the preset requirements. The training results of RBF and ELMAN are in figures 6, 7 and the average errors of the four neural networks are shown in table 3 .

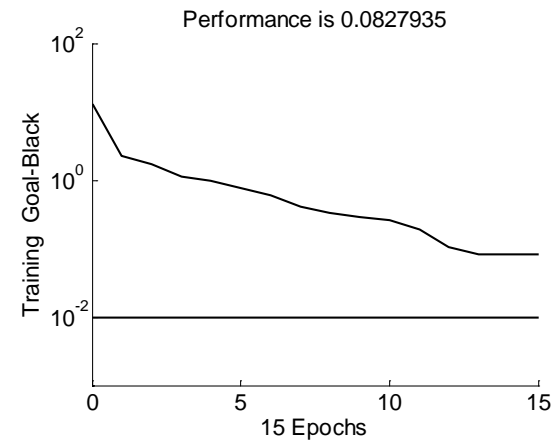

Fig. 6. Training result of $\mathrm{BP}$

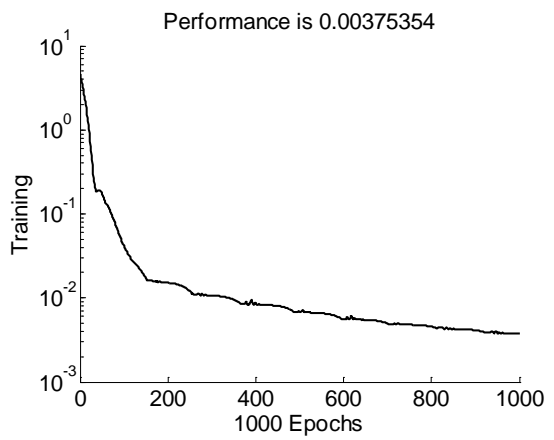

Fig. 7. Training result of ELMAN 
Tab. 3. Error comparison of the four neural networks

\begin{tabular}{cccccc}
\hline & $C$ & $D$ & $E$ & $G$ & $t_{\mathrm{d}}$ \\
\hline BP & 0.216 & 0.1 & 0.136 & 0.129 & 0.089 \\
RBF & 0.165 & 0.08 & 0.092 & 0.122 & 0.077 \\
GRNN & 0.226 & 0.177 & 0.188 & 0.202 & 0.181 \\
ELMAN & 0.212 & 0.12 & 0.122 & 0.197 & 0.101 \\
\hline
\end{tabular}

As shown in table 3, the comparison of the average error shows RBF neural network has minimum error in predicting the paddy drying outputs in deep fixed-bed, which means it has good performance in actual application.

\section{CONCLUSIONS}

Since paddy drying in deep fixed-bed is a complex process effecting by different factors, The effect rules of paddy drying in the base of quadratic orthogonal rotation combination design has been researched. To predict the paddy rice status parameters, different ANN have been built based prediction models, including BP, RBF, GRNN, ELMAN. The experimental results show that the training errors have all reached preset requirements. The prediction accuracy of BPNN is highest and GRNN is the lowest. The results lay foundations for the prediction of paddy drying and provide references for modeling the multi-parameter systems.

\section{References}

1. HUANG He, BAI Jicheng, LU Zesheng, GUO Yong-feng. Electrode Wear Prediction in Milling Electrical Discharge Machining Based on Radial Basis Function Neural Network [J]. Journal of Shanghai Jiaotong University(Science), 2009, 14(6): 736-741.

2. CHANG Lixia, PAN Ruru, GAO Weidong. Fashion Color Forecasting by Applying an Improved Back Propagation Neural Network [J]. Journal of Donghua University(English Edition), 2013, 30(1): 58-62.

3. HE Yongxiu, HE Haiying, WANG Yuejin, LUO Tao. Forecasting model of residential load based on general regression neural network and PSO-Bayes least squares support vector machine [J]. Journal of Central South University of Technology, 2011, 18(4): 1184-1192.

4. QU Hua, MA Wentao, ZHAO Jihong, WANG Tao. Prediction Method for Network Traffic Based on Maximum Correntropy Criterion [J]. China Communications, 2013(1): 134-145.

5. GAO Qian, YAN Weiwu, SHAO Huihe. Regularized RBF Network-Based Inferential Sensor and Its Application in Product Quality Prediction [J]. Acta Simulata Systematica Sinica, 2005, 17(7): 1609-1612, 1678.

6. ZHANG QH, SIMON X YANG. Prediction of performance indices and optimal of rough rice drying using neural networks [J]. Biosystems Engineering, 2002, 83(3): 281-290.

7. ZHENG Xianzhe, ZHOU Xiuli, XIA Jiqing. The study on drying condition influcing paddy mill quality [J]. Journal of Northeast Agricultural University, 2001, 32(01): 48-52. (in Chinese with English abstract)

8. WANG Dan-yang, LI Cheng-hua. Experiment Study on Influence of Drying Parameters on Drying Duration of Paddy Rice in a Deep Fixed-bed. Journal of Shenyang Agricultural University, 2008, 39(2): 213-217.

9. Qinghua Zhang, Simon X. Yang, Gauri S. Mittal, etc. Prediction of Performance Indices and Optimal Parameters of Rough Rice Drying using Neural Networks [J]. Biosystems Engineering , 2002, 83 (3), 281-290.

10. Brahim Doymaz, Mebmet Pala. The thin-layer drying characteristics of corn [J]. Journal of food engineering, 2003, (60): 125-130.

11.Li Ping, Zeng Lingke, Shui Anze, Jin Xueli, Liu Yanchun, Wang Hui. DESIGN OF FORECAST SYSTEM OF BACK PROPAGATION NEURAL NETWORK BASED ON MATLAB [J]. Computer Applications and Software, 2008, 25(4): 149-185. (in Chinese with English abstract)

12.ZHAO Chuang, LIU Kai, LI Diansheng. Freight Volume Forecast Based on GRNN [J]. 
Journal of The China Railway Society, 2004, 26(1): 12-15. (in Chinese with English abstract)

13. WEI Jinyan, RU Feng. Forecasting the Traffic Volume by the Model of GRNN and Studing It's Realization[J]. Journal of Changsha Communications University, 2006, 22(2): 46-50. (in Chinese with English abstract)

14. LIN Hongri. Forecasting Safety Stock of Supply Chain Using Artificial Neural Network [J]. Journal of Fuzhou Teachers College, 2004, 25(2): 72-75. (in Chinese with English abstract)

15. $\mathrm{T}$ Yacinoz, U Eminoglu. Short term and medium term power distribution load forecastingby neural networks [J]. Enegry Conversion and Manaegment, 2005, 46: 139-314.

16. Kamyar Movagharnejad.Maryam Nikzad Model ing of tomato drying using artificial neural.network [J]. Computers and Electronics in Agriculture, 2007, 59: 78-85.

17. Beigi M, Torki-Harchegani M, Mahmoodi-Eshkaftaki M. Prediction of paddy drying kinetics: a comparative study between mathematical and artificial neural network modelling[J]. 2016:39-39.

18. T Yacinoz,U Eminoglu.Short term and medium term power distribution load forecasting by neural networks[J]. Enegry Conversion and Manaegment, 2005,46:139-314.

19. Kamyar Movagharnejad.Maryam Nikzad Model ing of tomato drying using artificial neural. Network[J]. Computers and Electronics in Agriculture 2007,59:78-85.

20. Li J, Xiong Q, Wang K, et al. A Recurrent Self-Evolving Fuzzy Neural Network Predictive Control for Microwave Drying Process[J]. Drying Technology, 2016, 34(12):1434-1444.

21.Pati J R, Dutta S, Eliaers P, et al. Experimental Study of Paddy Drying in a Vortex Chamber[J]. Drying Technology, 2016(9).

22. Firouzi S, Alizadeh M R, Haghtalab D. Energy consumption and rice milling quality upon drying paddy with a newly-designed horizontal rotary dryer[J]. Energy, 2016.

23. Lilhare S F, Bawane N G. Artificial Neural Network Based Control Strategies for Paddy Drying Process[J]. International Journal of Information Technology \& Computer Science, 2014, 6(11).

24. Mittal G S; Zhang J (2000). Prediction of temperature and moisture content of frankfurters during thermal processing using neural network. Meat Science, 55, 13-24.

25. Fortuna L, Giannone P, Graziani S, Xibilia MG. Virtual Instruments Based on Stacked Neural Networks to Improve Product Quality Monitoring in a Refinery [J]. Instrumentation and Measurement, IEEE Transactions on, 2007, 56(1):95-101.

26. Golpour I, Chayjan R A, Parian J A, et al. Prediction of Paddy Moisture Content during Thin Layer Drying Using Machine Vision and Artificial Neural Networks[J]. Journal of Agricultural Science \& Technology, 2015. 